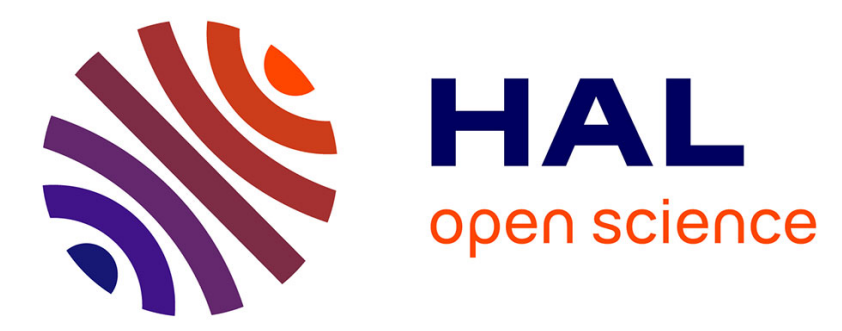

\title{
Detection of a southern peak in Mercury's sodium exosphere with the TNG in 2005
}

V. Mangano, François Leblanc, C. Barbieri, S. Massetti, A. Milillo, Gabriele Cremonese, C. Grava

\section{- To cite this version:}

V. Mangano, François Leblanc, C. Barbieri, S. Massetti, A. Milillo, et al.. Detection of a southern peak in Mercury's sodium exosphere with the TNG in 2005. Icarus, 2009, 201 (2), pp.424. 10.1016/j.icarus.2009.01.016 . hal-00533501

\section{HAL Id: hal-00533501 https://hal.science/hal-00533501}

Submitted on 7 Nov 2010

HAL is a multi-disciplinary open access archive for the deposit and dissemination of scientific research documents, whether they are published or not. The documents may come from teaching and research institutions in France or abroad, or from public or private research centers.
L'archive ouverte pluridisciplinaire HAL, est destinée au dépôt et à la diffusion de documents scientifiques de niveau recherche, publiés ou non, émanant des établissements d'enseignement et de recherche français ou étrangers, des laboratoires publics ou privés. 


\section{Accepted Manuscript}

Detection of a southern peak in Mercury's sodium exosphere with the TNG in 2005

V. Mangano, F. Leblanc, C. Barbieri, S. Massetti, A. Milillo, G. Cremonese, C. Grava

PII: $\quad$ S0019-1035(09)00037-2

DOI: $\quad$ 10.1016/j.icarus.2009.01.016

Reference: $\quad$ YICAR 8897

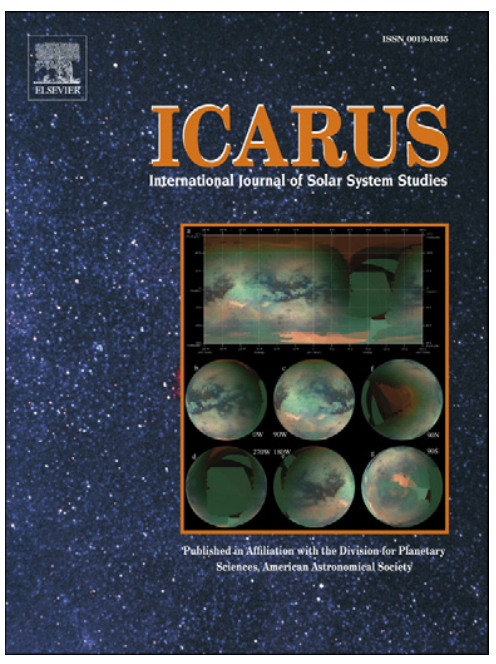

To appear in: Icarus

Received date: 18 November 2008

Revised date: 27 January 2009

Accepted date: 29 January 2009

Please cite this article as: V. Mangano, F. Leblanc, C. Barbieri, S. Massetti, A. Milillo, G. Cremonese, C. Grava, Detection of a southern peak in Mercury's sodium exosphere with the TNG in 2005, Icarus (2009), doi: 10.1016/j.icarus.2009.01.016

This is a PDF file of an unedited manuscript that has been accepted for publication. As a service to our customers we are providing this early version of the manuscript. The manuscript will undergo copyediting, typesetting, and review of the resulting proof before it is published in its final form. Please note that during the production process errors may be discovered which could affect the content, and all legal disclaimers that apply to the journal pertain. 
1

2

3

4

5

6

7
Corresponding author:

Valeria Mangano

INAF-Istituto di Fisica dello Spazio Interplanetario, via Fosso del Cavaliere 100, 00133 Roma, Italy, tel: +39 0645488 752, fax: +39 0649934386 , email: valeria.mangano@ifsi-roma.inaf.it 
2 Abstract

3

A long term plan of observations of the sodium exosphere of Mercury began in 2002 by using the high resolution echelle spectrograph SARG and a devoted sodium filter at the $3.5 \mathrm{~m}$ Galileo National Telescope (TNG) located in La Palma, Canary Islands. This program is meant to investigate the variations of the sodium exosphere appearance under different conditions of observations, namely Mercury's position along its orbit, phase angle and different solar conditions, as reported by previous observations in August 2002 and August 2003 (Barbieri et al., 2004 and Leblanc et al., 2006).

11 Here we present the analysis of data taken in June $29^{\text {th }}$ and $30^{\text {th }}$ and in July $1^{\text {st }} 2005$, when 12 Mercury's True Anomaly Angle (TAA) was in the range 124-130 . The spectra show particularly 13 intense sodium lines with a distinctive peak in emission localized in the southern hemisphere at 14 mid-latitudes. This seems to be a persistent feature related to consecutive favourable IMF 15 conditions inducing localized enhancements of surface sodium density. The comparison with 16 previous data taken by Potter et al. (2002) evidences a surprising consistency of the anti-sunward 17 component, which appears to remain constant regardless of the changing illumination and space 18 weather conditions at Mercury. 


\section{Introduction}

In 1974 the existence of a thin atmosphere around Mercury was discovered by Mariner 10, which revealed UV emissions of three atomic elements: H, He and O (Broadfoot et al., 1976). Three other elements ( $\mathrm{Na}, \mathrm{K}$, and $\mathrm{Ca}$ ) were later discovered with ground-based observations in the visible spectral range (Potter \& Morgan 1985, Potter \& Morgan 1986, and Bida et al. 2000, respectively). Due to the low surface number density (approximately $n=10^{5}-10^{7}$ atoms $/ \mathrm{cm}^{3}, P=$ $10^{-12}$ bar, on the dayside), this atmosphere can be considered collisionless. Therefore, the whole atmosphere is comparable with an exosphere having the exobase coincident with the planet's surface, i.e. a surface bounded exosphere (Stern, 1999).

More than thirty years after its discovery, the dynamics of such exosphere, the source and sink mechanisms and its relationship with solar activity, are still not clearly understood. Although NASA MESSENGER has already reached Mercury's orbit and detected gases (including Na) in the exospheric tail (McClintock et al., 2008), Earth-based observations are the only way to provide continuous data and to support exospheric simulations, and still remain an effective method to study and try to understand the Hermean environment.

Therefore, a campaign of observations started in 2002 at the Telescopio Nazionale Galileo (TNG) with the goal to provide the largest possible database of $\mathrm{Na}$ exosphere by covering a full Mercury's year at different true anomaly angles (TAA), phase angles and solar conditions. The observations were performed with the TNG high resolution echelle spectrograph SARG, equipped with a narrow filter of $60 \AA$ centred on the sodium doublet at 5890-5896 $\AA$ (D lines), in order to suppress the adjacent spectral orders. The projected slit is 26.7 arcseconds long and 0.4 arcseconds wide. Table 1 gives the main parameters of the spectroscopic configuration; more detailed information on SARG can be found at the website: http://www.pd.astro.it/sarg/.

The observations performed in 2002 at TAA $171-174^{\circ}$ and in 2003 at TAA $163-168^{\circ}$ have been published by Barbieri et al. (2004; hereafter Paper I) and Leblanc et al. (2006; hereafter Paper II). Paper I showed the capability of SARG/TNG to detect Mercury's Na exosphere and reported column density values consistent with previous observations (Killen and Ip, 1999) as well as with model simulations by Leblanc and Johnson (2003). Paper II reported about three nights of observations, two of which with a remarkably stable Na exosphere; the third one showed a localized feature in the early morning varying on a very short time scale.

In the present paper we describe the observations performed in 2005 and the main remarkable features identified in Mercury's exosphere (Sections 1 and 2). Then we discuss (Section 
13 ) and conclude (Section 4) on the possible origins of these features within the context of what is

2 known on the sources of Mercury's sodium exosphere.

3

4

6 .

\section{Observations}

In 2005 observations were performed during three evenings: June $29^{\text {th }}$ ant $30^{\text {th }}$, and July $1^{\text {st }}$, just before and soon after the sunset, until the moment in which the telescope safety mode blocked the observation, at Mercury's elevation of $13.5^{\circ}$ above the horizon. At such large airmasses (2-4), large values of the seeing are expected. In the present paper seeing values are given as one $\sigma$ of equation (1) in Sprague et al. (1997). These numbers should therefore be multiplied by 1.67 to obtain the FWHM seeing values used in many publications on Mercury's sodium exosphere (Potter et al. 2006; 2007; 2008). The seeing $1 \sigma$ values for the three nights were in the range $1.5-2.0$ " in June $29^{\text {th }}, 0.9-1.3^{\text {" }}$ in June $30^{\text {th }}$ and $1.5-2.1$ " in July $1^{\text {st }}$.

To save time for observations, the read-out time was reduced by binning the CCD in the spatial direction ( $2 \mathrm{x}$ binning). As a consequence, the effective spatial pixel was $0.16 \times 2=0.32$ arcseconds. Due to the composition of the Earth's and Mercury's orbits inclinations plus the Mercury's axis inclination, the slit was placed at an angle of $11.2-12.4^{\circ}$ with respect of the E-W direction (with the Sun being to the W). The planet disk was 6.7-7.0 arcseconds in diameter, and more than $50 \%$ of it was illuminated by sunlight. Dawn terminator was visible (as in 2002 and 2003 observations). Mercury's main parameters are listed in Table 2.

We collected six useful spectra during the first night, thirteen during the second and five during the third night. The exposure time was 120 seconds for all the spectra. The peculiarity of this data set with respect to the previous ones of 2002 and 2003 is the much stronger sodium emission and, consequently, the high values of the ratios between exospheric sodium emission and sky background intensity. These ratios range from 2.4 to 14 for the first night, from 2.5 to 140 for the second, and from 28 to 60 for the third night. The occurrence for such remarkable enhancement is probably due to the conjunction of several reasons: clear sky conditions, a closer position of Mercury to the Sun and, most important, a larger radial heliocentric velocity of Mercury, which increases the solar flux effects and, hence, raises the excitation of the D1 and D2 resonant transitions.

\section{Data Analysis}


The extraction procedure of exospheric sodium emission is the same that was used for the 2003 data set (Paper II); hence, here it will be repeated just briefly. The preliminary treatment consists of flat field, bias and sky subtraction. Spectral calibration is obtained by comparing the spectra with a Th-Ar reference lamp spectrum.

A noticeable improvement with respect to the previous data sets is a more accurate sky subtraction: special care was taken, in fact, to avoid subtracting any sodium at the edge of the slit. Such a procedure allows us to retain the faint $\mathrm{Na}$ emission present also in the regions out of the disk and, in particular, in the anti-sunward direction up to $\sim 2 \mathrm{R}_{M}$ from the planet surface.

The main points of the reduction phase are summarized in the following:

- G-factors: they are two values related to the photon efficiency in the process of resonant scattering producing the $\mathrm{Na}$ exospheric emission (Brown and Yung, 1976). They can be calculated by assuming a constant solar flux at the D wavelengths during the three nights. Values of g-factors, seeing (both conventional seeing and seeing sigma) and Doppler shifts (in $\mathrm{m} \AA$ ) are given in Table 3 .

- Continuum: its estimation is performed close to the D emission lines. In order to convert instrumental counts (ADUs) into Rayleigh (R) brightness (the Rayleigh unit is a measure of the omni-directional emission rate in a column of unit area along the line of sight, with $1 \mathrm{R}$ $=10^{6}$ photon $/ \mathrm{cm}^{2} / \mathrm{s}$; Chamberlain and Hunten, 1987), a reflectivity model developed by Hapke (1986) and adapted to Mercury by Sprague et al. (1997) is used to derive the solar flux reflected by Mercury's surface. The comparison between this model and the measured continuum provides the conversion factors (from now on addressed as 'calibration factors') needed to calibrate the exospheric emission. This approach to calibrate Mercury's exospheric emission has been largely used in previous publications (Leblanc et al., 2006; Potter et al., 2006). In particular, it allows to get rid of the atmospheric absorption by using Mercury's surface as a calibrated candle and, therefore, to compare observations obtained at different air mass values. In addition, the comparison between the continuum and the Hapke surface reflectivity model provides an estimation of the seeing because the model depends on both the geometry and the seeing value at the time of the observations. Therefore, a good fit between the continuum and the Hapke model is also an indication of a good choice of the seeing value during our observations.

- Slit positioning above Mercury's disk: this is a delicate point. The correct position of the slit in the North-South (N-S) direction (that is, perpendicularly to the slit) is roughly derived by comparing the instrumental theoretical position with a picture of the planet reflected by the 
mask containing the slit. The correct position of the slit in the East-West (E-W) direction, instead, is derived by superposing the maximum of the measured continuum over the maximum of the calculated Hapke model. As in Paper II, the uncertainty related to the instrument pointing is estimated to be 0.5 ".

- Finally, the extraction of the exospheric emission: it is performed by using three different methods: (1) a Voigt profile to fit the D1 and D2 solar Fraunhofer lines (Hummer, 1962), as in the 2003 data set (Paper II); (2) a solar spectrum properly Doppler-shifted to simulate both the continuum and the Fraunhofer lines, and, (3) finally, thanks to its peculiar position on one side of the broad Fraunhofer absorption, a linear interpolation of the solar line in the interested region (as in Paper I). The comparison among the three cited methods shows no significant difference. Hence, linear interpolation was chosen as being the most straightforward solution, and is applied to all the spectra of the three nights.

The results in column density $\left(\right.$ atoms $/ \mathrm{cm}^{2}$ ) are obtained with the assumption that D1 is optically thin and then by following the conversion described by Kameda et al. (2007).

The errors on the final values of column density have three different origins: (1) the instrumental noise; (2) the error caused by the extraction of the exospheric emission with a linear interpolation, and (3) the errors introduced by the conversion from ADUs to Rayleigh and from Rayleigh to atoms $/ \mathrm{cm}^{2}$. The instrumental noise and the error of extraction are negligible with respect to the errors due to the calibration. Since the Hapke model cannot be applied outside Mercury's visible disk, only calibration factors for the spectra placed on it can be considered. The plot in Fig. 1 (for the second night), shows that all calibration factors are close to $2.45 \pm 0.70$ for the spectra placed on the disk. Moreover, no significant dependence of these calibration factors with respect to airmass can be noticed. Therefore, a mean value can be assumed as the best value to be applied to all spectra of each night. We derived an average calibration factor of 1.92 for the first night, 2.45 for the second and 2.02 for the third. The first and the third values are very close (about $5 \%$ of difference), while the second one appears to be larger by about $20 \%$, which highlights the significant better atmospheric conditions during this night of observation.

\section{FIGURE 1}

Regarding the error in the conversion from ADU to Rayleigh, which is mostly due to the calibration factor, we derive an estimate of $35 \%$. When the conversion to atoms $/ \mathrm{cm}^{2}$ is applied, the contribution of the g-factor uncertainty should be considered, and the total uncertainty in column density calculation rises to $46 \%$. For this uncertainty is really high, we decided to stop our calculation to brightness values (Rayleigh). 


\section{Data Interpretation/Discussion}

The Figs. 2, 3 and 4 show the final images of the measured emission for the three different nights of observation superposed to the planet disk.

During the first night we sampled the exosphere at almost regular distances between the different slit positions, while during the second night the larger number of spectra led to an almost complete coverage of the sodium emission from the exosphere of the planet. Unfortunately, during the third night the coverage of the mid-latitude regions (at both hemispheres) was very poor, even if a general trend of the spatial distribution of the exospheric emission can be deduced as well.

The column density values are in good agreement with previous measurements $\left(1-3 \cdot 10^{11}\right.$ atoms $/ \mathrm{cm}^{2}$ with respect of $2.8-3.8 \cdot 10^{11}$ by Killen et al., 1990) and with the predictions of current exospheric models (Leblanc and Johnson, 2003; Mura et al., 2007).

Our observations reveal a constant pattern during the three nights, characterized by enhancements along the equator and a peak in the mid-latitude southern hemisphere (the peak being less evident during the first night).

\section{FIGURES 2, 3, 4}

The equatorial enhancements have been already observed in the past (Potter et al., 2003; Killen et al., 2001) and seem to validate the theories of a major role in the exospheric production played by thermal desorption (TD) and photon-stimulated desorption (PSD) (McGrath et al. 1989; Hunten and Sprague, 1997). Both mechanisms act at their maximum in the sub-solar regions, whereas also atmospheric blurring would move the brightness peak toward the visible disk (Potter et al., 2006).

However, the observed maximum at mid-latitudes in the southern region cannot be explained by a uniform exosphere dominated by PSD or TD, and could be related, instead, to a local peak of release of the sodium trapped in the surface. This peak is clearly visible on the second night, when a better coverage of the planet is available. During this night (June $30^{\text {th }}$ ) the peak brightness is almost $15 \%$ higher than the average peak brightness of the other spectra (this is probably due to the better seeing conditions), while it is $12 \%$ for the third night, and $10 \%$ for the first. The occurrence of this peak during all the three nights, always in the same region of the disk with a regular profile both in the D2 and D1 component along the slits (i.e. the spatial dimension), is a strong indication in the direction of the real existence of the peak. 
The possible explanations of this feature could be: (1) a localized sodium-rich region in the surface, and/or (2) a local peak of efficiency of one or more sodium release processes. In the first case, the sodium-rich region should be within $260-300^{\circ} \mathrm{E}$ longitude and $10-40^{\circ} \mathrm{S}$ latitude. This region is not included in any of the radar bright spots detected by Sprague et al. (1998) as having higher abundances of sodium bearing minerals, and denoted as $\mathrm{A}\left(15^{\circ} \mathrm{E}, 25^{\circ} \mathrm{S}\right), \mathrm{B}\left(15^{\circ} \mathrm{E}, 55^{\circ} \mathrm{N}\right)$ and $\mathrm{C}\left(120^{\circ} \mathrm{E}, 15^{\circ} \mathrm{N}\right)$. However, Harmon et al. (2007) refer of a fainter radar feature located at $18^{\circ}$ E, $27^{\circ} \mathrm{S}$ which could be probably related to the Bartok crater and that may be associated to the observed emission peak, if we take into account the seeing effect (seeing sigmas between 0.3 and 0.6 Hermean radii).

Localized emission peaks have been already observed in the past, at both hemispheres at mid-latitudes (see Potter et al., 2006 for a review; and Leblanc et al., 2008 for latest detection). Following these authors, the observed higher emission could be related to the interplanetary space conditions and in particular to the sign of the $\mathrm{B}_{\mathrm{x}}$ component of the Interplanetary Magnetic Field (IMF), the $\mathrm{x}$ component being in the planet-Sun direction. In fact, the inter-connection between the Hermean magnetic field and the IMF may allow solar wind particles to enter the cusps down to the surface, and reconnection is virtually always present at Mercury apart from peculiar cases in which the $\mathrm{B}_{Z}$ component is dominant (e.g. see Fig. 5 of Massetti et al., 2007). A realistic IMF configuration with significantly $\mathrm{B}_{\mathrm{X}}>0(<0)$ causes reconnection mainly in the southern (northern) dayside magnetopause, with a consequent particle precipitation which could in turn cause an intense surface release due to ion-sputtering and, hence, a southern (northern) localized enhancement of atoms in the exosphere. The magnetic reconnection induced by IMF $\mathrm{B}_{\mathrm{X}}$ can be amplified by a negative $B_{Z}$ component, as well as by an increased solar wind density (Massetti et al., 2007).

\section{FIGURES 5, 6}

Given the impossibility to have direct information on the IMF at Mercury, we try to derive them by comparing the space weather condition at the Earth (detected by the ACE satellite, located at the L1 Sun-Earth Lagrangian point) with the solar coronal magnetic field as extrapolated from photospheric measurements (Wilcox Solar Observatory magnetograms). In this way, we find that, taking into account the time shift to the orbit of Mercury, the Sun was relatively active in the period of our observations, leading to several interplanetary perturbations (Figs. 5 and 6).

The ACE data reveals that an interplanetary discontinuity occurred between 182-183 DOY $\left(\mathrm{DOY}=\right.$ day of the year, corresponding to July $1^{\text {st }}$ and $\left.2^{\text {nd }}\right)$, marked by an increase of the solar wind density (up to about $50 \mathrm{~cm}^{-3}$ ) which was shortly followed by a steep increase of both temperature (up to $\sim 5 \times 10^{5} \mathrm{~K}$ ) and speed (up to $650 \mathrm{~km} / \mathrm{s}$ ) (Fig. 5). The event was likely caused by the transit of 
1 June $30^{\text {th }}$ (180 DOY, SoHO/EIT data) and represented by the black pattern in Fig. 6 (bottom) above

2 the image of the solar magnetic field. By taking into account the position of both the Earth and

3 Mercury with respect to the Sun $\left(180^{\circ}\right.$ and $120^{\circ}$ solar longitude, respectively), the solar rotation and

4 the relative time lag due to the propagation speed of the interplanetary perturbation, we can expect

5 that the fast stream probably reached the inner planet in the middle of 176 DOY (June $25^{\text {th }}$ ), that is

6 about 4 days before our TNG ground-based observations (180-182 DOY). As ACE data show, at

7 the Earth orbit the fast stream was followed by a long period (6-7 days) characterized by a slightly

8 positive ( $\sim 2 \mathrm{nT}$, on the average) IMF $\mathrm{B}_{\mathrm{X}}$ component. By scaling back this condition to $0.42 \mathrm{AU}$ we could expect an IMF $\mathrm{B}_{\mathrm{X}} \sim 6-12 \mathrm{nT}$ at Mercury (the IMF strength at Mercury's orbit being about 3-6 times larger than at 1 AU, e.g.: Burlaga, 2001), that lasted for the whole observation period. This

11 IMF configuration is consistent with an asymmetric reconnection pattern with a large open cusp 12 region in the southern hemisphere, where most of the solar wind plasma precipitation takes places, 13 peaking at mid-latitudes (Massetti et al. 2007). A fraction of that ion flux $(\approx 10 \%)$ is expected to be 14 able to reach the dayside planetary surface, contributing to the release of sodium directly via ion15 sputtering, or indirectly by enhanced diffusion of sodium from interior hence increasing the photonstimulated desorption rate (Potter, 1995; Killen et al., 2004; Mura et al., 2008).

On the other hand, the analysis of the solar coronal magnetic field, computed from the observed photospheric field (WSO), does not completely match the IMF $\mathrm{B}_{\mathrm{X}}$ time evolution. In fact, by using the position of the coronal hole to synchronize the solar wind and coronal field data sets (see Fig. 6), we found that after the transit of the fast stream associated with the coronal hole and embedded in a negative magnetic sector (IMF $\mathrm{B}_{\mathrm{X}}>0$ ), there is a net transition to a large positive magnetic sector, leading a negative IMF $\mathrm{B}_{\mathrm{X}}$. This means that the IMF configuration changed significantly while the solar wind travelled till the Earth (ACE), and that somewhere $\mathrm{B}_{\mathrm{X}}$ changed its sign. Hence, on the base of the available observations it cannot be clearly stated if the IMF $\mathrm{B}_{\mathrm{X}}$ sign at Mercury during the period of observation was positive or negative. To try solve this ambiguity we inspected the Ecliptic-plane IMF forecast, computed by the Geophysical Institute (University of Alaska Fairbanks) on the basis of the Hakamada-Akasofu-Fry (HAF) solar wind model (Fry et al., 2001): Fig. 7 shows that the IMF likely had a 4 sector configuration, with the Earth embedded in a negative IMF $\mathrm{B}_{\mathrm{X}}$ (away from the Sun), while Mercury was inside the adjacent positive IMF $\mathrm{B}_{\mathrm{X}}$ sector (toward the Sun). The computed IMF geometry then confirms the sector structure observed by ACE spacecraft (Fig. 6, top panel): and we can be sufficient confident that the IMF $\mathrm{B}_{\mathrm{X}}$ component was mainly positive during the period our observations, leading to a magnetic merging dominating in the southern hemisphere. 
Moreover, by inspecting the SOHO/LASCO CME catalogue (available on the website: http://cdaw.gsfc.nasa.gov/CME_list/) we find that during our $\mathrm{Na}$ observations, several Coronal Mass Ejections (CMEs) occurred close to ecliptic plane and to the east limb of the Sun, likely triggered by the active region AR0871 that was approximately facing Mercury. CMEs are violent ejections of plasma from the solar corona characterized by a steep increase in plasma density, temperature and velocity. They also drag typical magnetic field structures known as "magnetic clouds", characterised by twisted field lines. Some solar flares also occurred in the same period. They are sudden and intense brightness enhancements occurring in the solar corona, and emitting radiation (with energies of the order of $10^{27} \mathrm{erg} / \mathrm{s}$ ) as well as particles, including heavy ions. Flares occur in active regions, like the one that was facing Mercury. However, the intensity of flares (detected by GOES satellite located around the L1 point too) which occurred between June $26^{\text {th }}$ and July $1^{\text {st }}$ is always sensibly moderate (between C and B class).

By inspecting the emission brightness values during the three nights, June $30^{\text {th }}$ appears to be globally stronger in emission with respect to the other two. The total enhancement is estimated to be $19 \%$ higher than in the third night. Unfortunately, the conversion error from ADU to Rayleigh accounts for $35 \%$ uncertainty on the measured emission brightness. Hence, we cannot state whether the observed enhancement is a real feature in the present observation, or not. However, should it resemble a real global enhancement, the present feature could be caused by a higher efficiency of all the release processes (or of the dominant ones) or by a higher efficiency of the resonant scattering process producing the exospheric D lines. Both these explanations would be reasonable if a higher solar flux reached Mercury between the first and the second night of observation. In fact, higher plasma density, velocity and $\mathrm{He} / \mathrm{H}$ ratio are expected to cause a higher rate of efficiency of the release processes, leading to a global enhancement in the exospheric emission that could be consistent with the observations.

Finally, as already mentioned in Section 2, the accurate extraction of sodium emission in all the pixels of the spectra shows evidence of a sodium component out of the disk in the anti-sunward direction. The Mercury sodium tail was discovered by Potter et al. (2002) and observed by other authors (Baumgardner et al., 2008; Kameda et al., 2008; Potter and Killen, 2008). This feature is clearly visible in the spectra that lie completely outside the disk, but similar information can be extracted from the spectra crossing the disk as well, although the shadow of the planet itself decreases the efficiency of resonant scattering and, hence, the 'visibility' of the exospheric sodium component. All our spectra sample the sodium tail up to $\sim 2 \mathrm{R}_{\mathrm{M}}$ far from the planet surface. Potter et al. (2002) plotted the sodium tail up to the surface along the axis of the tail, i.e. in the anti-sunward 
$1 \quad\left(\mathrm{TAA}=114^{\circ}\right)$ and May $26^{\text {th }} 2001\left(\mathrm{TAA}=125^{\circ}\right)$. We compare our data with Potter et al. one by 2 averaging the values of the spectra at same $\mathrm{x}$-value (Figs. 2, 3 and 4) and by considering the 3 different sizes of the planet disk at the different dates. To account for the different size of Mercury 4 at the time of observations, we compare our data $E$ ' to a reference observation $E$, by multiply $E$ ' by 5 a factor $D^{\prime 2} / D^{2}$ (where $D$ and $D^{\prime}$ are the disk diameters during $E$ and $E$ ' measurements, 6 respectively). Moreover, we have to convert the measured distances into heliocentric distances 7 (Potter et al., 2002), due to the different phase angles of the Earth with respect of Mercury and the 8 Sun.

\section{FIGURE 8}

Figure 8 shows the results of the comparison of our three nights (solid lines) with the Potter et al. observations (filled triangles). In all cases the tail emissions have similar shape and brightness. This is consistent with the similar orbital position of Mercury during all the compared five nights (TAAs are in the range $114^{\circ}-130^{\circ}$ ). In fact, it is expected that the sodium tail varies along the orbit of Mercury due to the solar radiation pressure variation, which is a function of both the heliocentric distance and the radial velocity (Potter and Morgan, 1987; Potter and Killen, 2008). Figure 8 also shows similar shape and brightness of the disk emissions during the first and third nights as compared to June $5^{\text {th }} 2000$ data, even if the greater seeing of July $1^{\text {st }}$ causes broadening of the emission profile for this night. May $26^{\text {th }} 2001$ data, instead, appear to be higher (by a factor 1.7 in intensity of the maximum) when compared with June $30^{\text {th }}$, our most intense night. Even if considering all the discrepancies, our comparison with Potter et al. (2002) provides a rather and surprisingly good agreement of the 'tail' emission. Actually, the comparison of the sodium tail measured on May $26^{\text {th }} 2001$ with our measurement on June $29^{\text {th }} 2005$ displays a very good agreement. This is the best agreement and actually it is the case where the two profiles have been obtained for very close TAA. This result suggests, therefore, that the shape and brightness of the tail depend essentially on the position of Mercury along its orbit and does not depend significantly on the mechanism leading to the ejection of the sodium atoms. If confirmed by further observations, it would imply that the main population of Mercury's tail is produced by a mechanism not strongly dependent on the solar wind conditions (the most variable mechanism of ejection, in particular from solar minimum to maximum conditions).

\section{Conclusions}

In this paper we report the observation of Mercury sodium exosphere performed at TNG during three nights (June $29^{\text {th }}-$ July $1^{\text {st }}, 2005$ ) at sunset. We observe column density values above 
1 the disk in the range $1-3 \cdot 10^{11}$ atoms $/ \mathrm{cm}^{2}$, with a peak of emission in the southern hemisphere at 2 mid-latitudes.

Higher emissions have been already correlated to solar activity (Killen et al. 2001), while asymmetries have been explained by models simulating the interaction between solar plasma and planetary magnetic field considering the different reconnection geometries driven by the IMF $\mathrm{B}_{\mathrm{X}}$ component (Sarantos, 2001; Kallio and Janhunen, 2003). Hence, we try to correlate the observed features with solar activity by inspecting the SWO solar data, ACE solar wind data and SOHO/LASCO CMEs data at L1, and GOES flares data from a geostationary orbit. SWO evidences an active region and $\mathrm{ACE}$ data show that its effects remain for a long period (6-7 days) with a positive IMF $\mathrm{B}_{\mathrm{X}}$ component (scaled at Mercury to $\sim 6-12 \mathrm{nT}$ ). Moreover, many CMEs and flares occurred in the Sun in the direction of Mercury in the days before our observations, leading to the conclusion that some SEP events should have occurred and that a period of medium or high level space weathering affected the Hermean environment just before and during our observation run. Moreover, the modelled IMF geometry and the ACE spacecraft data, supports the hypothesis that the IMF $\mathrm{B}_{\mathrm{X}}$ component was consistent with reconnection in the southern hemisphere.

The comparison of our data with previous one at similar TAA (June $5^{\text {th }} 2000$, and May $26^{\text {th }}$ 2001; see Potter et al., 2002) reveals consistency both in profiles and intensities. In particular, the substantial invariability of the shape and brightness of the sodium emission in the anti-sunward direction for similar conditions of observation suggests that the tail surprisingly displays rather constant characteristics and is therefore only slightly dependent on other conditions than Mercury's TAA. This conclusion need further investigations and long-period observations, but it clearly illustrates that key information on Mercury's exosphere might be obtained by observing Mercury's sodium tail even close to its disk.

\section{Acknowledgements}

Based on observations made with the Italian Telescopio Nazionale Galileo (TNG) operated on the island of La Palma by the Fundación Galileo Galilei of the INAF (Istituto Nazionale di Astrofisica) at the Spanish Observatorio del Roque de los Muchachos of the Instituto de Astrofisica de Canarias.

This work was partly supported by the Italian Space Agency ASI, the INAF-IFSI Institute in Rome, and by the EUROPLANET network.

Wilcox Solar Observatory data used in this study are is currently supported by NASA and was obtained via the web site http://wso.stanford.edu by courtesy of J.T. Hoeksema. 
We thank the ACE SWEPAM and MAG instrument teams and the ACE Science Center for 2 providing the ACE data (available on the website: http://www.srl.caltech.edu/ACE/ASC/level2/). 3 


\section{References}

Barbieri, C., Verani, S., Cremonese, G., Sprague, A., Mendillo, M., Cosentino, R., Hunten, D., First observations of the Na exosphere of Mercury with the high-resolution spectrograph of the 3.5M Telescopio Nazionale Galileo, Planet. Sp. Sci. 52, 1169-1175, 2004

Baumgardner, J., Wilson, J., Mendillo, M., Imaging the sources and full extent of the sodium tail of the planet Mercury, Geo. Res. Lett., v.35, L03201, 2008

Bida, T.A., Killen, R.M., Morgan, T.H., Discovery of calcium in Mercury's atmosphere, Nature, Volume 404, Issue 6774, pp. 159-161, 2000

Broadfoot, A.L., Shemansky, D.E., Kumar, S., Mariner 10 - Mercury atmosphere, Geo. Res.Lett., vol. 3, Oct. 1976, p. 577-580, 1976

Brown, R.A., Yung, Y.L., Io, its atmosphere and optical emissions, In: Jupiter, University of Arizona Press, p. 1102-1145, 1976

Burlaga L.F., Magnetic fields and plasmas in the inner heliosphere: Helios results, Planet. Sp. Sci., vol. 49, Issue 14-15, p. 1619-1627, 2001

Chamberlain, J.W., Hunten, D.M., Theory of planetary atmospheres: an introduction to their physics and chemistry, Academic Press, Inc. (International Geophysics Series. Volume 36), 1987

Domingue, D.L., Sprague, A.L., Hunten, D.M., Dependence of Mercurian atmospheric column abundance estimations on surface-reflectance modeling, Icarus, vol. 128, Issue 1, pp. 75-82, 1997

Fry, C.D., Sun, W., Deehr, C. S., Dryer, M., Smith, Z., Akasofu, S.-I., Tokumaru, M., Kojima, M, Improvements to the HAF solar wind model for space weather predictions, J. Geoph. Res., vol. 106, Issue A10, pp. 20985-21002, 2001

Hapke B., Bidirectional reflectance spectroscopy. IV - The extinction coefficient and the opposition effect, Icarus, vol. 67, p. 264-280, 1986

Hunten D. M., Sprague A. L., Origin and character of the lunar and Mercurian atmospheres, Adv. Sp. Res., vol. 19, p. 1551, 1997

Hummer D.G., Non-coherent scattering: I. The redistribution function with Doppler broadening, R. Astron. Soc. Month. Not., vol. 125, p. 21-37, 1962

Kameda, S., Yoshikawa, I., Ono, J., Nozawa, H., Time variation in exospheric sodium density on Mercury, Planet. Sp. Sci.,vol. 55, Issue 11, p. 1509-1517, 2007

Kameda, S., Kagitani, M., Okano, S., Yoshikawa, I., Ono, J., Observation of Mercury's sodium tail using Fabry-Perot Interferometer, Adv. Sp. Res., vol. 41, Issue 9, pp. 1381-1385, 2008

Killen, R.M., Potter, A.E., Morgan, T.H., Spatial distribution of sodium vapor in the atmosphere of Mercury, Icarus, 85(1), p.145, 1990 
Killen, R.M., Ip, W.H., The Surface-bounded Atmospheres of Mercury and the Moon, Rew. Geoph., vol.37, n.3, p361-406, 1999

Killen, R.M., Potter, A.E., Reiff, P., Sarantos, M., Jackson, B.V., Hick, P., Giles, B., Evidence for space weather at Mercury, J. Geoph. Res., vol. 106, Issue E9, pp. 20509-20526, 2001

Killen R. M., Sarantos M., Potter A. E., Reiff, P., Source rates and ion recycling rates for Na and K in Mercury's atmosphere, Icarus, vol. 171, Issue 1, pp. 1-19, 2004

Leblanc F., Doressoundiram A., Schneider N., Mangano V., López Ariste A., Lemen C., Gelly B., Barbieri C., Cremonese G., High latitude peaks in Mercury's sodium exosphere: Spectral signature using THEMIS Solar Telescope, Geophys. Res. Lett., 35, L18204, doi:10.1029/2008GL035322, 2008

Leblanc, F., Barbieri, C., Cremonese, G., Verani, S., Cosentino, R., Mendillo, M., Sprague, A., Hunten, D., Observations of Mercury's exosphere: Spatial distributions and variations of its Na component during August 8, 9 and 10, 2003, Icarus, vol 185, Issue 2, pp. 395-402, 2006

Leblanc, F., Johnson, R.E., Mercury's sodium exosphere, Icarus, Volume 164, Issue 2, pp. 261-281, 2003

Massetti S., Orsini S., Milillo A., Mura A., Modelling Mercury's magnetosphere and plasma entry through the dayside magnetopause, Planet. Sp. Sci., vol. 55, pp. 1557-1568, 2007

McClintock, W.E., $\quad$ Bradley, E.T., $\quad$ Vervack, R.J., $\quad$ Killen, R.M., $\quad$ Sprague, A.L., Izenberg, N.R., Solomon, S.C., Mercury's Exosphere: Observations During MESSENGER's First Mercury Flyby, Science, vol. 321, Issue 5885, pp. 92 , 2008

McGrath, M.A., R.E. Johnson and L.J. Lanzerotti, Sputtering of sodium on the planet Mercury, Nature 323, 694, 1986

Mura, A., Milillo, A., Orsini, S., Massetti, S., Numerical and analytical model of Mercury's exosphere: Dependence on surface and external conditions, Planet. Sp. Sci., vol. 55, Issue 11, p. 1569-1583, 2007

Mura A., Wurz P., Lichtenegger H.I.M., Schleicher H., Lammer H., Delcourt D., Milillo A., Orsini S., Massetti S., Khodachenko M.L., The sodium exosphere of Mercury: Comparison between observations during Mercury's transit and model results, submitted to Icarus, 2008

Potter, A.E., Morgan, T.H., Variation of sodium on Mercury with solar radiation pressure, Icarus, vol. 71, pp. 472-477, 1987

Potter, A.E., Morgan, T.H., Discovery of sodium in the atmosphere of Mercury. Science 229, pp. 651-653, 1985

Potter, A.E., Morgan, T.H., Potassium in the atmosphere of Mercury, Icarus, vol. 67, pp. 336-340, 1986 
Potter, A.E., Chemical sputtering could produce sodium vapor and ice on Mercury, Geoph.

2 Res. Lett., vol. 22, Issue 23, pp. 3289-3292, 1995

3

Potter, A.E., Killen, R.M., Morgan, T.H., The sodium tail of Mercury, Meteoritics \&

4 Planetary Science, vol. 37, no. 9, pp. 1165-1172, 2002

5 Potter, A.E., Killen, R.M., Morgan, T.H., Solar radiation acceleration effects on Mercury

6 sodium emission, Icarus, vol. 186, Issue 2, pp. 571-580, 2007

7

8 Issue 1,pp. 1-12, 2008

Sprague, A.L., Schmitt, W.J., Hill, R.E., Mercury: Sodium Atmospheric Enhancements,

10 Radar-Bright Spots, and Visible Surface Features, Icarus 136, I. 1, pp. 60-68, 1998

Stern, S.A., The lunar atmosphere: history, status, current problems, and context, Rev.

12 Geophys., vol. 37, No. 4, pp. 453 - 491, 1999 


\section{Table and Figure Captions}

$$
\text { Table } 1 \text { - SARG main parameters. }
$$

Table 2 - Mercury's parameters at observation time.

Table 3 - Seeing values, radial velocity shifts on spectra and g-factors for the three nights.

Figure 1 - Plot of the calibration factor versus airmass values $(\mathrm{Nb}$. time and slit numbers increase to the right) for the night of June $30^{\text {th }}, 2005$. Only the slits that cross the disk (hence, for $46,47,50,53,54,57,58,59$ and 60) show an almost constant value around 2.20. An average value equal to 2.45 was calculated.

Figure 2 - Global view of the observations performed during the first night (June $29^{\text {th }}$ July $1^{\text {st }}$ ) for the D1 lines in emission brightness $(\mathrm{kR})$. Colour bars on the right give the corresponding emission brightness (in kiloRayleigh), while seeing sigma are expressed on top (in Mercury radii units). Numbers of the slit are also put on the figures to give the time sequence of the observation during each night (increasing number corresponds to increasing time with $\sim 3$ minutes of delay between two consecutive slit positions). Sun is on the right side (hence, this is also the illuminated side of the disk); North Celestial Pole and Mercury North Pole are on top of the figure. Six slits were available, showing higher brightness in the illuminated side of the disk, with a slightly higher value (more extended to higher latitudes) in the Southern hemisphere.

Figure 3 - Global view of the observations performed during the second night (June $30^{\text {th }}$ ) for the D1 lines in emission brightness (kR). Same descriptions as previous figure. Thirteen slits were available, almost all on the disk, with an almost total coverage of it that evidences a more evident and localized enhancement in the Southern hemisphere.

Figure 4 - Global view of the observations performed during the third night (July $\left.1^{\text {st }}\right)$, for D1 lines in emission brightness (kR). Same descriptions as previous figure. Five slits were available with a poor coverage of the interest region in the South, but still an enhancement is clearly visible. 
Figure 5 - ACE data of IMF $B_{X}$ and $B_{Z}, H e / H$ ratio, proton temperature $\left(T_{P}\right)$, density $\left(n_{P}\right)$ and velocity $\left(\mathrm{v}_{\mathrm{P}}\right)$ between doy 170-191 of 2005. A fast stream arrival is recognizable by the increase of the solar wind velocity at the end of DOY 182 and is evidenced by a vertical line. A peak in all these parameters is clearly present. Note that during the fast stream passage, the $\mathrm{B}_{\mathrm{X}}$ component is $>0$.

Figure 6 - The IMF BX component measured by ACE (second plot from the top in Figure 5) is reported, with time and y-axis reversed. Bottom left: large scale solar magnetic field as extrapolated at $20 \mathrm{R}_{\odot}$ (coronal field) from SOHO data. On June $28^{\text {th }} 2005$ Earth is at $180^{\circ}$ (central vertical line), solar equator is the horizontal line at mid altitude, while Mercury position is at about $120^{\circ}$. Light gray region is for IMF $\mathrm{B}_{\mathrm{X}}<0$, dark gray for IMF $\mathrm{B}_{\mathrm{X}}>0$. The solid black pattern in the middle of the figure shows the position of the coronal hole from which the fast stream originated (visible at the Earth from DOY 182). The effect of solar rotation leads to observe back in the past as we move to the right and to the future as we move to the left. Hence, we can compare the evolution of the $\mathrm{B}_{\mathrm{X}}$ component at the Earth (upper panel) to the correspondent IMF $\mathrm{B}_{\mathrm{X}}$ at the Sun by checking the main features, i.e. the sign inversion (solid arrows). Note that in the solar magnetic field the IMF $B_{X}$ during the fast stream is $>0$, but there is a sign inversion after about four days, which is not present in the $\mathrm{B}_{\mathrm{X}} \mathrm{ACE}$ data (first arrow to the left).

Figure 7 - IMF geometry on the ecliptic plane as computed by the Geophysical Institute (University of Alaska Fairbanks). Red regions are for IMF away from the Sun (IMF $\mathrm{B}_{\mathrm{X}}<0$ ), blue ones for IMF toward the Sun (IMF $B_{X}>0$ ). During the days of interest Mercury (black dot with denoted with ' $M$ ') lies permanently inside IMF $B_{X}$ positive region.

Figure 8 - Comparisons of each night of TNG observation (average of the central slits, crossing the equator) with the tail profile of Potter et al. (2002) for two nights: June $5^{\text {th }} 2000$, and May $26^{\text {th }} 2001$. Sun is on the right side and double $\mathrm{x}$-axis shows both the heliocentric distance and the distance expressed in Hermean radii $\left(\mathrm{R}_{\mathrm{M}}\right)$.Good agreement is displayed for all cases in the tail region (see semilog plot on the right side); good agreement is also shown in the first and third night with the data of June $5^{\text {th }} 2000$ on the disk region (central part, see normal plot on the left side). June $30^{\text {th }}$ instead shows higher values compared with this night, but not enough with respect to May $26^{\text {th }}$ 2001. Larger profile is shown on July $1^{\text {st }}$ due to higher seeing values. 
$1 \quad$ Figure 1

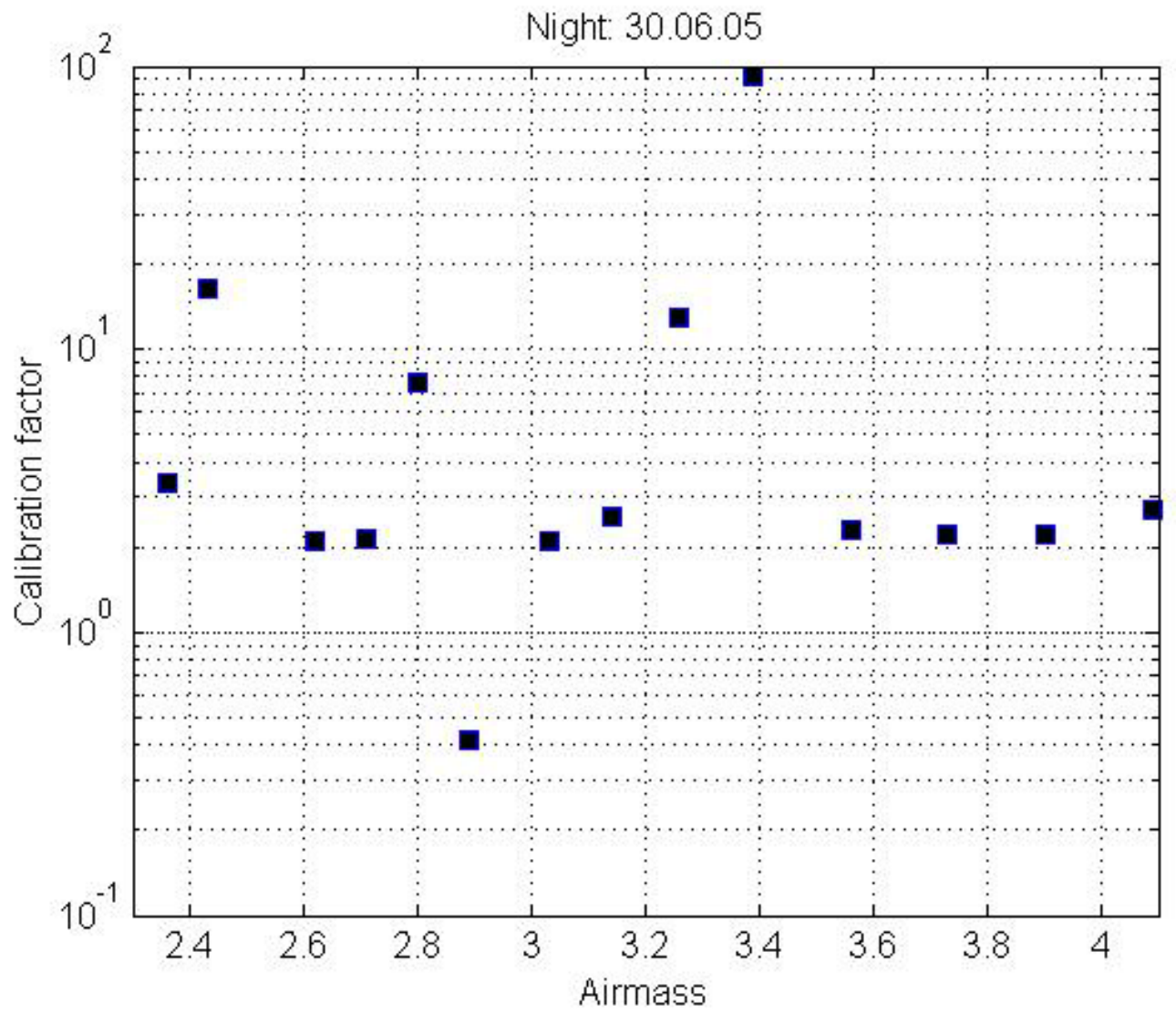

Figure 2

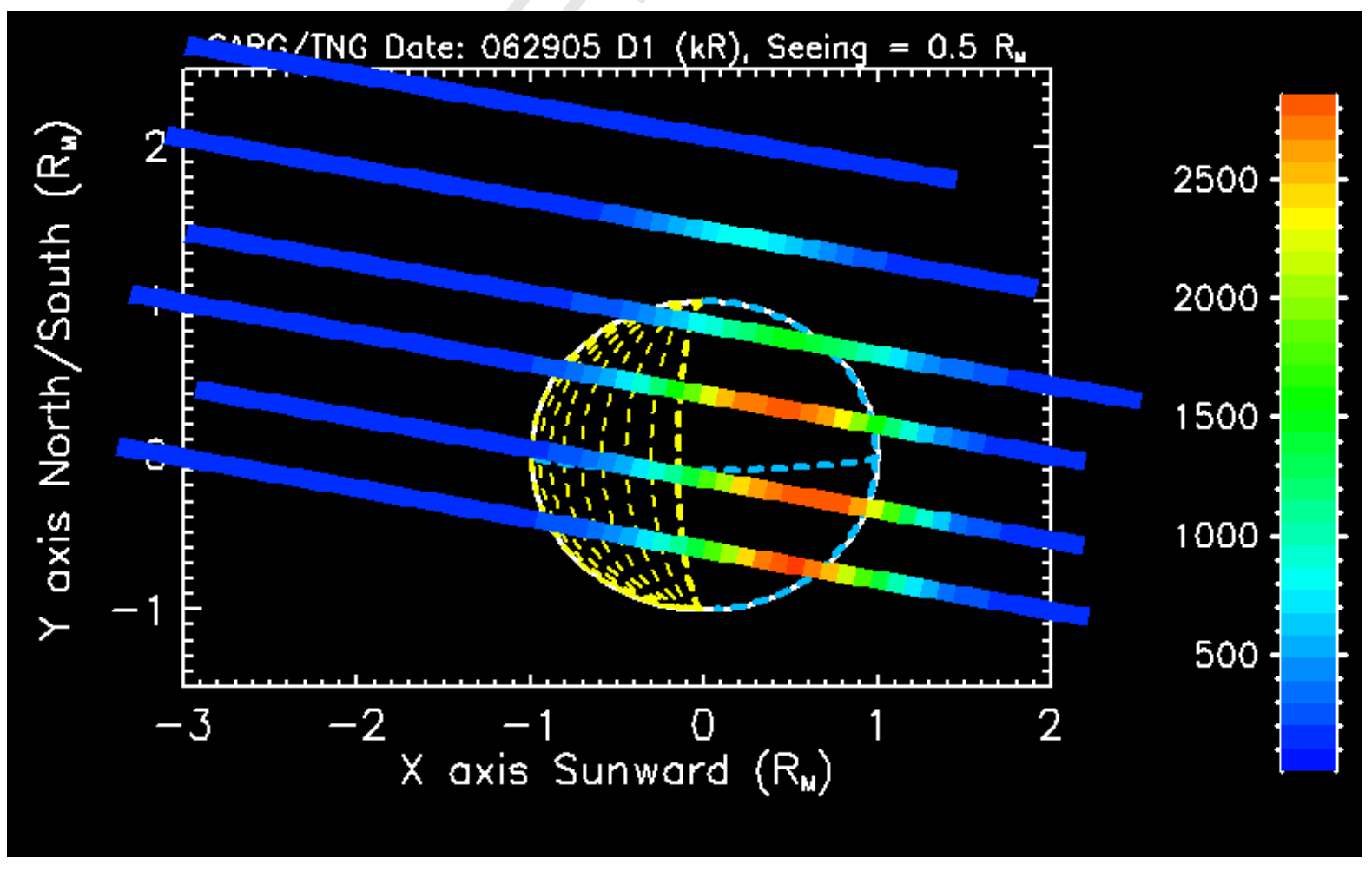




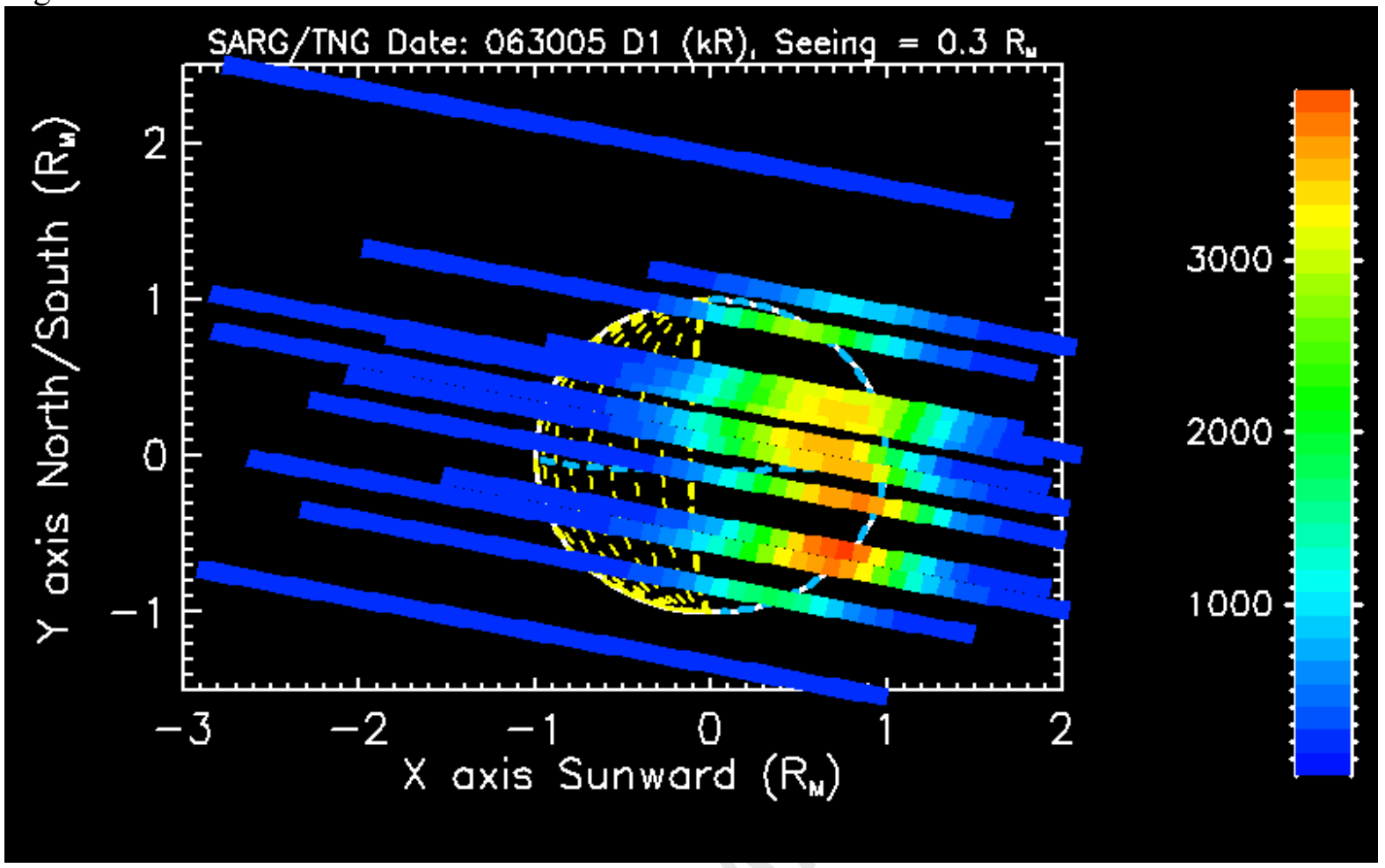

Figure 4

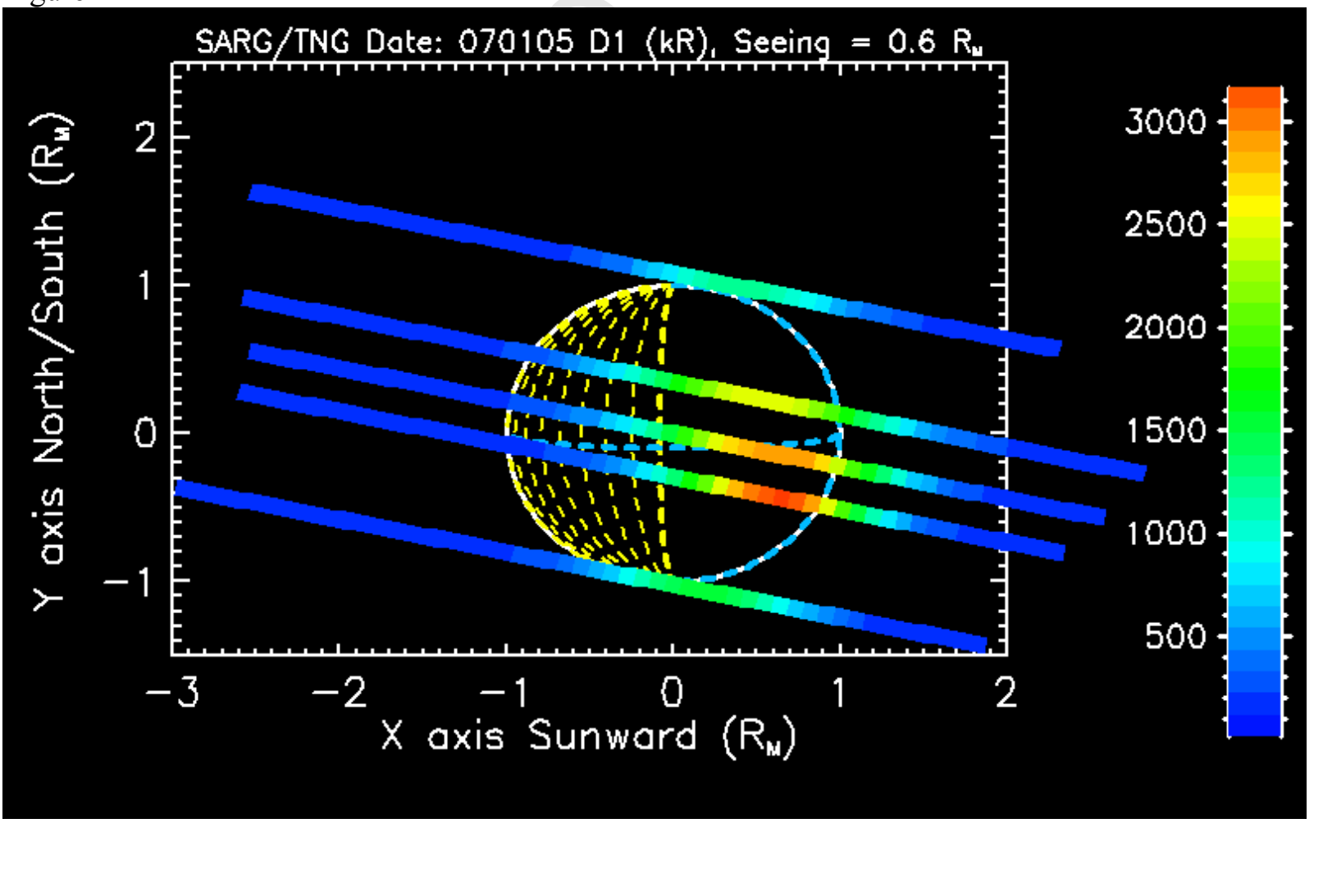


1 Figure 5

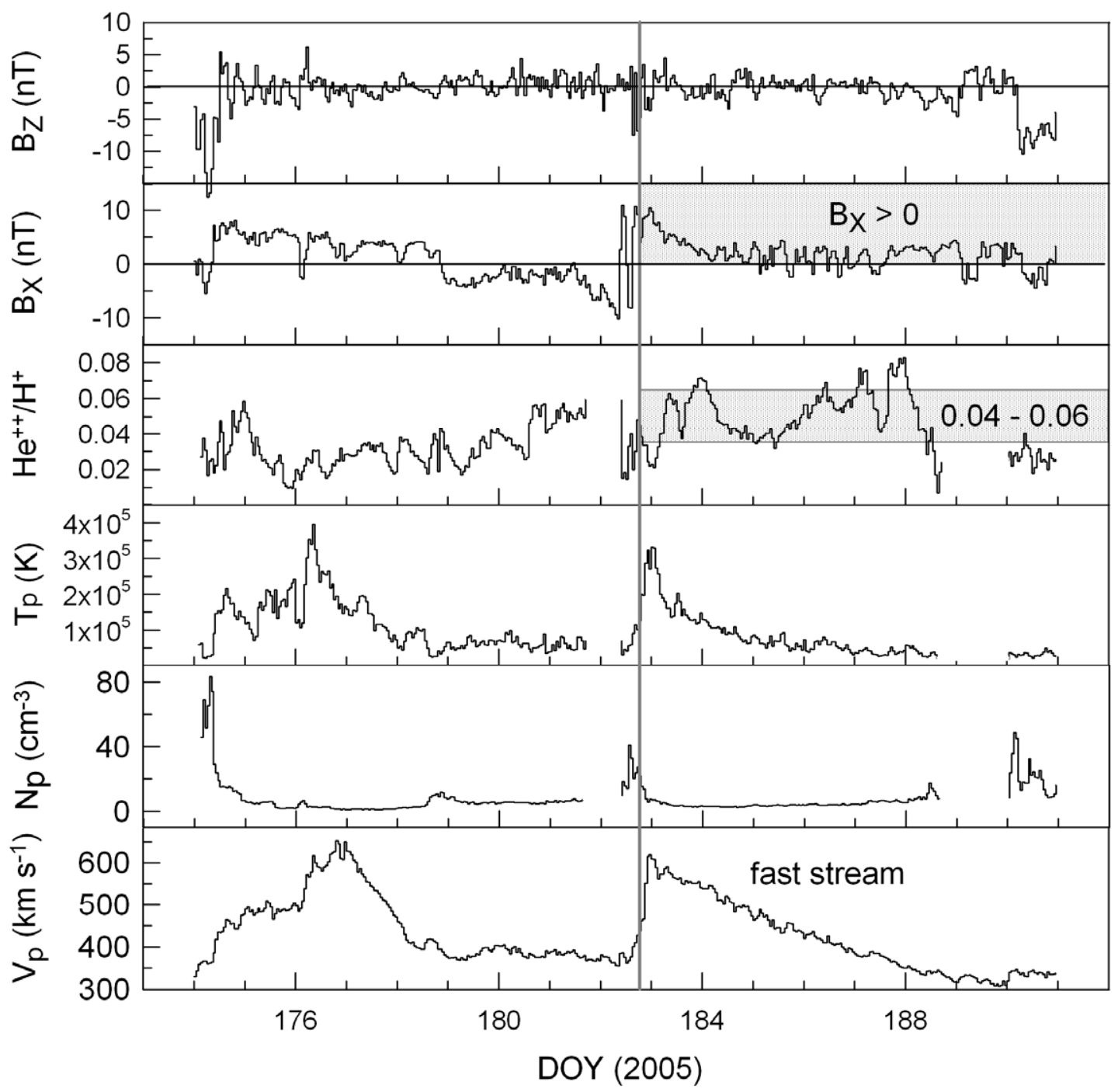

2
3 
Figure 6

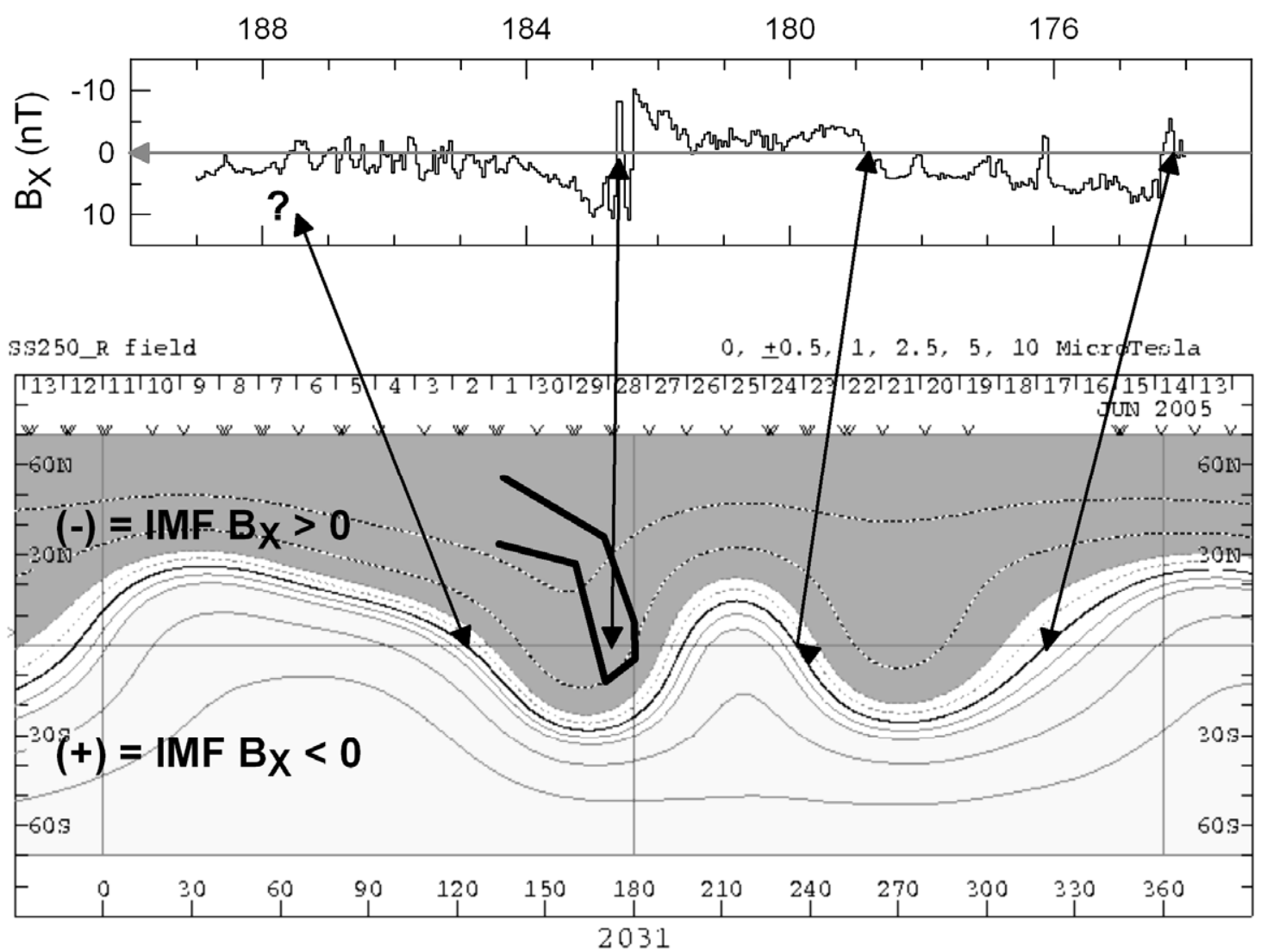

2 
Figure 7

2
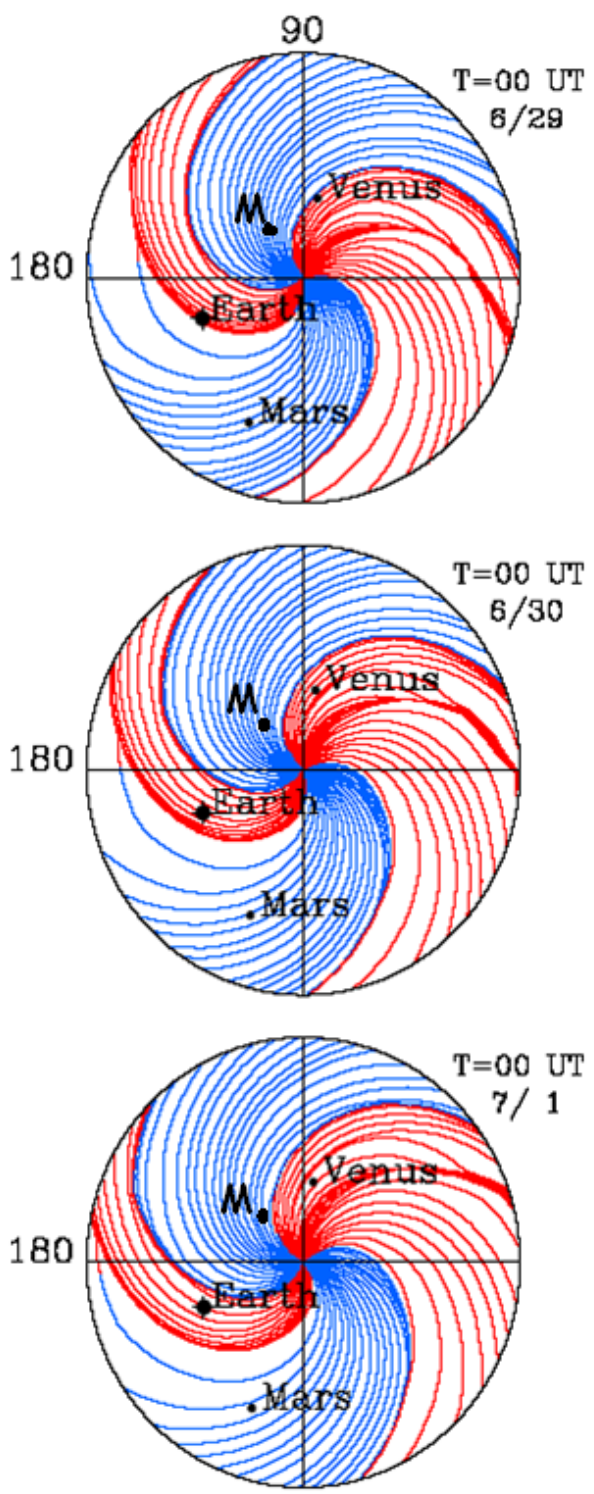

3

4 
Figure 8
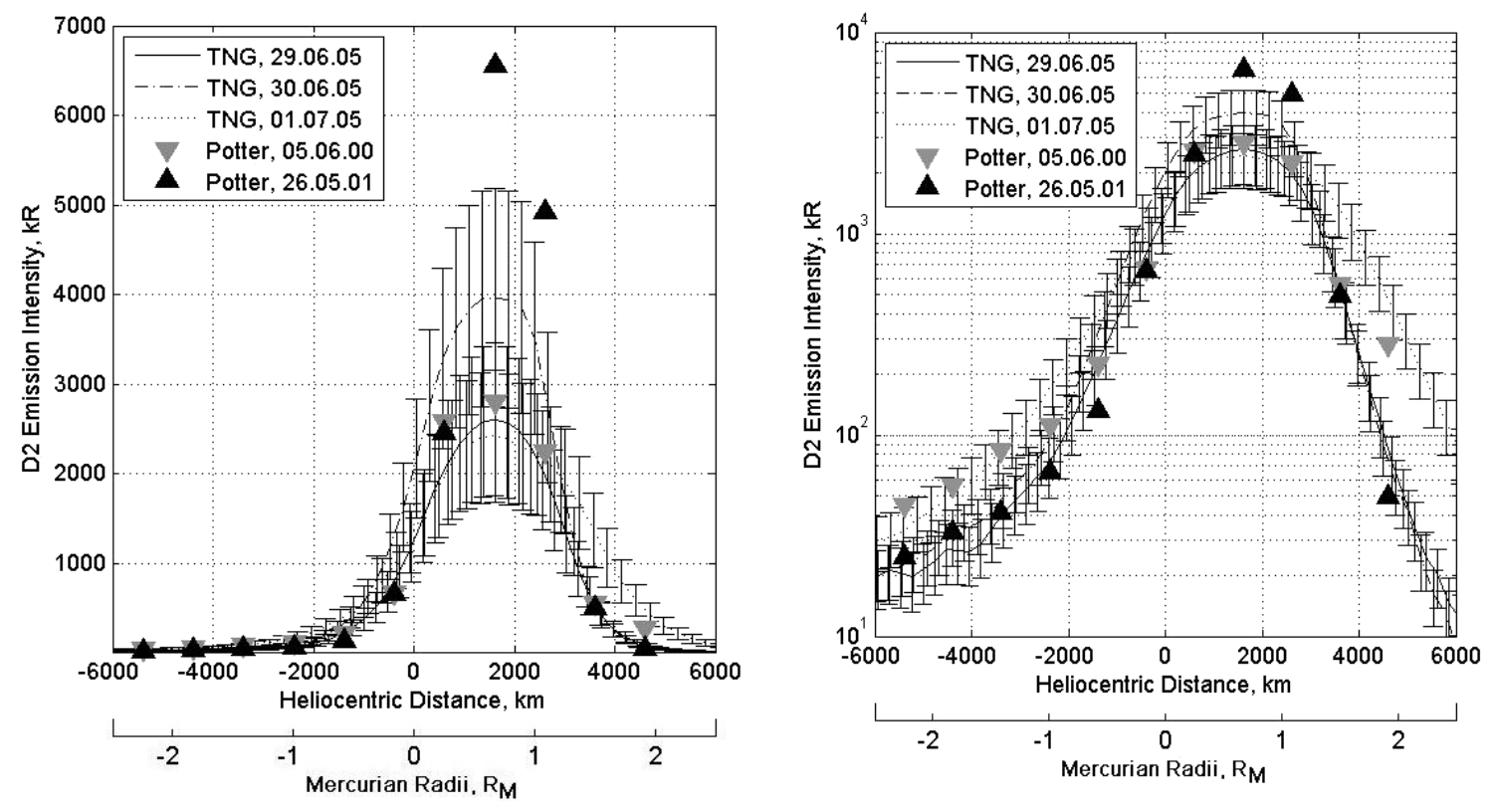

2 
1

2

3

4

5

6

7

8

9

10

11 Table 3

12

13

14

Table 1

Table 2

\section{0}

2

\begin{tabular}{|l|l|}
\hline Spectrograph resolution & 115000 \\
\hline Slit length and width & $26.7 \times 0.40$ arcsec \\
\hline Pixel dimension and scale & $0.022 \AA, 0.16$ arcsec \\
\hline CCD dimension & $2 \mathrm{~K} \times 4 \mathrm{~K}$ pixels \\
\hline
\end{tabular}

\begin{tabular}{|c|c|c|c|}
\hline Parameters & June $29^{\text {th }}$ & June $30^{\text {th }}$ & July $1^{\text {st }}$ \\
\hline Sun-Mercury Distance (AU) & 0.422 & 0.426 & 0.430 \\
\hline Illuminated Fraction & $57 \%$ & $56 \%$ & $54 \%$ \\
\hline Angular Diameter (arcsec) & 6.79 & 6.91 & 7.03 \\
\hline Mercury-Earth Radial Velocity & $-28.72 \mathrm{~km} / \mathrm{s}$ & $-28.58 \mathrm{~km} / \mathrm{s}$ & $-28.42 \mathrm{~km} / \mathrm{s}$ \\
\hline Mercury-Sun Radial Velocity & $8.12 \mathrm{~km} / \mathrm{s}$ & $7.77 \mathrm{~km} / \mathrm{s}$ & $7.39 \mathrm{~km} / \mathrm{s}$ \\
\hline Sun-Earth-Mercury Phase Angle & $81.37^{\circ}$ & $83.34^{\circ}$ & $85.28^{\circ}$ \\
\hline True Anomaly Angle (TAA) & $124^{\circ}$ & $127^{\circ}$ & $130^{\circ}$ \\
\hline Sub-Earth Point (West Long. and Lat.) & $246.9^{\circ}, 5.5^{\circ}$ & $242.0^{\circ}, 5.7^{\circ}$ & $237.3^{\circ}, 5.8^{\circ}$ \\
\hline Sub-Solar Point (West Long. and Lat.) & $328.4^{\circ}, 0.0^{\circ}$ & $325.5^{\circ}, 0.0^{\circ}$ & $322.6^{\circ}, 0.0^{\circ}$ \\
\hline
\end{tabular}

\begin{tabular}{|c|c|c|c|c|c|c|}
\hline Date & $\begin{array}{l}\text { Seeing } \\
\text { Sigma } \\
\text { (") }\end{array}$ & $\begin{array}{l}\text { Seeing } \\
\text { FWHM } \\
\text { (") }\end{array}$ & $\begin{array}{c}\text { Sun-Mercury } \\
\text { Radial velocity } \\
\text { (mÁ) }\end{array}$ & $\begin{array}{l}\text { Earth-Mercury } \\
\text { Radial velocity } \\
\text { (mÁ) }\end{array}$ & $\begin{array}{c}\text { g-factor } \\
\text { D2 }\end{array}$ & $\begin{array}{c}\text { g-factor } \\
\text { D1 }\end{array}$ \\
\hline $29 / 06 / 2005$ & $1.5-2.0$ & $2.5-3.3$ & 159 & -574 & 21.2 & 13.7 \\
\hline $30 / 06 / 2005$ & $0.9-1.3$ & $1.5-2.2$ & 152 & -562 & 19.2 & 12.6 \\
\hline $01 / 07 / 2005$ & $1.5-2.1$ & $2.5-3.5$ & 145 & -558 & 17.3 & 11.7 \\
\hline
\end{tabular}

